\title{
Analysis on the effect of Hoisting and Lowering Motions of Tower Cranes on Cargo Oscillation
}

\author{
Mingxiao Dong ${ }^{1, a}$, Haiping Dong ${ }^{2, b}$, Bo Pang ${ }^{1, c}$ and Jiyong Wang ${ }^{1, d}$ \\ ${ }^{1}$ Mechatronic Engineering School, Shandong Jianzhu University, Jinan, 250101, China \\ 2 Industrial Technology Department, Weihai Vocational College, Weihai, 264210, China

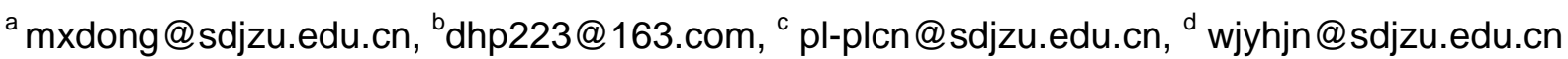

Keywords: Tower crane; cargo; Oscillation; Hoisting and lowering Motion.

Abstract. In order to find a method to suppress the cargo oscillation and precise positioning of tower cranes, this paper studied the effect of the lifting mechanism motion on the amplitude and frequency of cargo oscillation of the tower cranes. The calculation formulas of the amplitude attenuation of the cargo oscillation are derived that provides a theoretical basis for the design of anti-swing the controller.

\section{Introduction}

Tower Cranes has three kinds of basic working conditions including transition, rotation and hoisting movement. In order to improve the working efficiency, it usually carries out the three motions at the same time. This makes cargo movement more complex ${ }^{[1-3]}$. Namely, the cargoes do space pendulum with variable pendulum length and the suspension point moves along with the cargoes. The oscillation models are a two order nonlinear time varying differential equation. In order to realize the position control and anti-swing control, usually the nonlinear models are linearized into a simple pendulum motion, and then put forward the anti-swing control strategies. In this paper, the influence of the hoisting and lowering motion on the cargo oscillation is analyzed quantitatively and the action law of the hoisting and lowering motion on the amplitude and frequency of the cargo oscillation is deduced ${ }^{[4-5]}$.

\section{Dynamic Characteristics of Tower Cranes}

Tower cranes transport cargoes by the rotation of crane boom and trolley motion along the cargo boom. According to the motion characteristics, we set up a polar coordinate system $\left\{\mathrm{e}_{\rho}, \mathrm{e}_{\psi}\right\}$ whose coordinate origin is located at the intersection of the rotary center line of the tower body and the rotary surface of the crane cargo boom, non-inertial Cartesian coordinate system $\{\mathrm{i}, \mathrm{j}, \mathrm{k}\}$, and spherical coordinate $\operatorname{system}\left\{\mathrm{e}_{\theta}, \mathrm{e}_{\varphi}, \mathrm{e}_{l}\right\}$ whose coordinate origin located at the suspension point of the hoisting cable and the suspension point moves with the trolley and synchronously rotates with the cargo boom. The coordinate systems are shown in Fig. 1. 


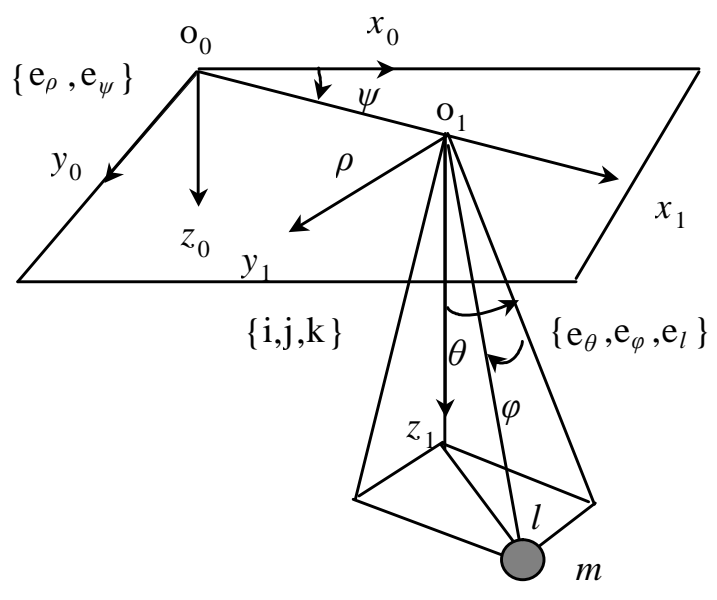

Fig. 1. Tower crane coordinate systems

The cargo hanging point in the polar coordinate systems is the position of $(\rho, \psi), \rho$ and $\psi$ respectively denote the displacement of the trolley and the rotation angle of the cargo boom. The positions of the cargo in the non-inertial spherical coordinates are described with the three generalized coordinates $(l, \theta, \varphi)$, where $l$ denotes the hoisting rope length, $\varphi$ denotes the angle between the hoisting rope and $x_{1} \mathrm{O}_{1} z_{1}$ plane, $\theta$ is the angle between the projection of the hoisting cable in $x_{1} \mathrm{o}_{1} z_{1}$ plane and plumb line through the cargo hanging point.

The nonlinear dynamic models of the crane are established according to the motion equation of the Lagrange-Euler with the transition, rotation and hoisting movements. Around the equilibrium state $\theta_{\mathrm{e}}=0^{\circ}$ and $\varphi_{\mathrm{e}}=0^{\circ}$, the linear models of the crane are as following.

The crane system has five parameters which are the trolley velocity \& or acceleration $\$$, the rotary angular velocity of the jib $\psi$ or the angular acceleration $\psi \ll$, cargo lifting velocity $\xi$, or acceleration $\&$, cargo sway angles $\theta$ and $\varphi$. In this paper, the first three variables are control variables and the latter two are controlled variables ${ }^{[6,7]}$.

According to the actual situation of the crane work, given the following assumptions and requirements: (1) the mass of the hoisting rope being located at the hook; (2) the hoisting rope stiffness is large enough, the length change is negligible; (3) ignoring air resistance. Given the $x_{1} \mathrm{O}_{1} z_{1}$ plane position potential energy is zero, the nonlinear dynamic models of the crane is established according to the motion equation of the Lagrange-Euler at the same time transition, rotation and hoisting movements. In the near equilibrium state $\theta_{\mathrm{e}}=0^{\circ}$ and $\varphi_{\mathrm{e}}=0^{\circ}$, namely the load swing angle less than $10^{\circ}$, linear models are obtained by applying a small disturb to the system, neglecting the higher order terms, and then keeping the first terms ${ }^{[8,9]}$. The linear models of the crane at the same time to make transition motion, rotation and hoisting movement are as following.

$$
\begin{aligned}
& M-M \rho \psi \delta-m g \theta+b_{\rho} \rho=F_{\rho} \\
& \left(J_{m}+M \rho^{2}\right) \psi+2 M \rho \rho \alpha \delta-m \rho g \varphi+b_{\psi} \psi \delta=F_{\psi} \\
& m(g)+b_{l} \& F_{l}
\end{aligned}
$$

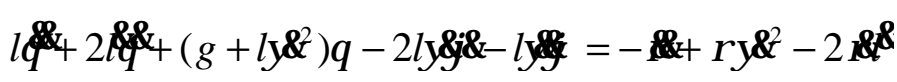

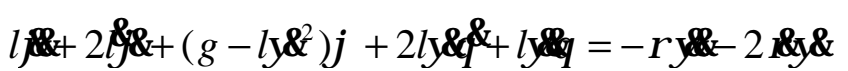

\section{Action Law of Hoisting and Lowering Motions on Load Swing}

It is usually considered that the hoisting and lowering motions of cranes have little effect on the cargo oscillation. In the case of ignoring the hoisting and lowering motion, the anti-sway control systems of the cranes are designed, so that the control accuracy can not realized. The hoisting and lowering 
motions make the tower crane system become as a weak damped two order oscillation system. And angle and angular velocity of the cargo oscillation are a periodic function whose amplitude and frequency are constantly changing. In the following discussion, the influence of the hoisting and lowering motions on the amplitude and frequency of the cargo oscillation is discussed.

The nature frequency in $\mathrm{e}_{\theta}$ direction is

$$
\omega_{\mathrm{n} 1}^{2}=(\mathrm{g}+h \psi \&) / l .
$$

The damping coefficient is

$$
\xi=\&\left(l \omega_{\mathrm{n} 1}\right) .
$$

The natural frequency in $\mathrm{e}_{\varphi}$ direction is

$$
\omega_{\mathrm{n} 2}^{2}=(\mathrm{g}-l \psi \&) / l .
$$

The damping coefficient is

$$
\xi=\&\left(l \omega_{\mathrm{n} 2}\right) .
$$

Because of $l \psi^{2}$ and $\mathrm{g}$ are small, $\omega_{\mathrm{n} 1}$ and $\omega_{\mathrm{n} 2}$ are approximately

$$
\omega_{\mathrm{n}}^{2}=\mathrm{g} / l \text {. }
$$

The oscillation frequency of cargo is

$$
\omega_{\mathrm{d}}=\omega_{\mathrm{n}} \sqrt{1-\xi^{2}} .
$$

The amplitude decay or increase of the amplitude in a swing period is

$$
\exp \left(-\pi \hat{\phi} \sqrt{\mathrm{g} l-l^{\&}}\right) .
$$

When lifting the cargo, the damping coefficient is negative value, the oscillation magnitude is increasing, and swing period is becoming smaller. On the contrary lowering the cargo, the damping coefficient is positive value, the oscillation is damped swing, and the swing period becomes bigger. Namely the hoisting and lowering motions change the amplitude and frequency of cargo oscillation. Fig. 2 shows the response curve of the cargo oscillation when the lifting weight respectively taking the hoisting motion into an account and ignoring the hoisting motion when hosting the cargo.

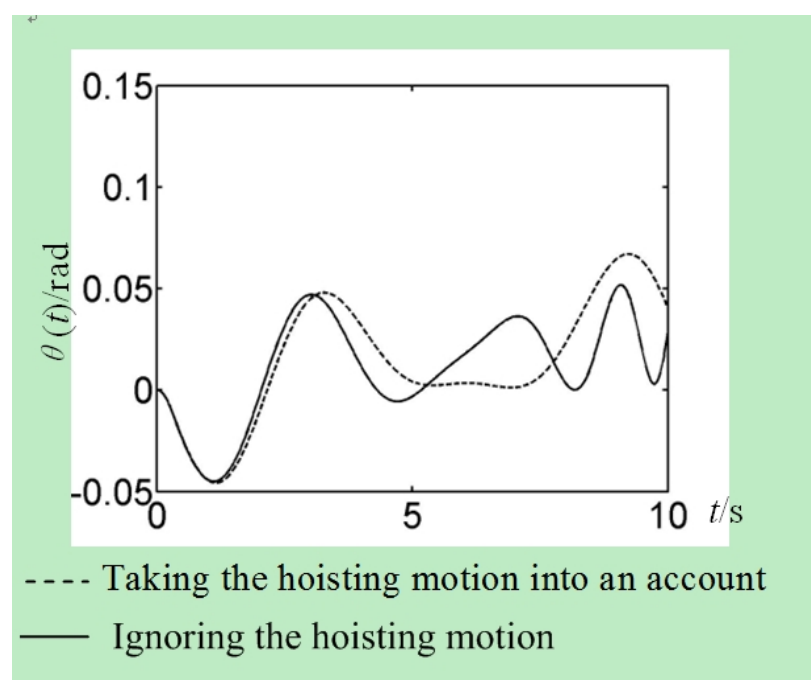

(a) Oscillation angle $\theta(t)$

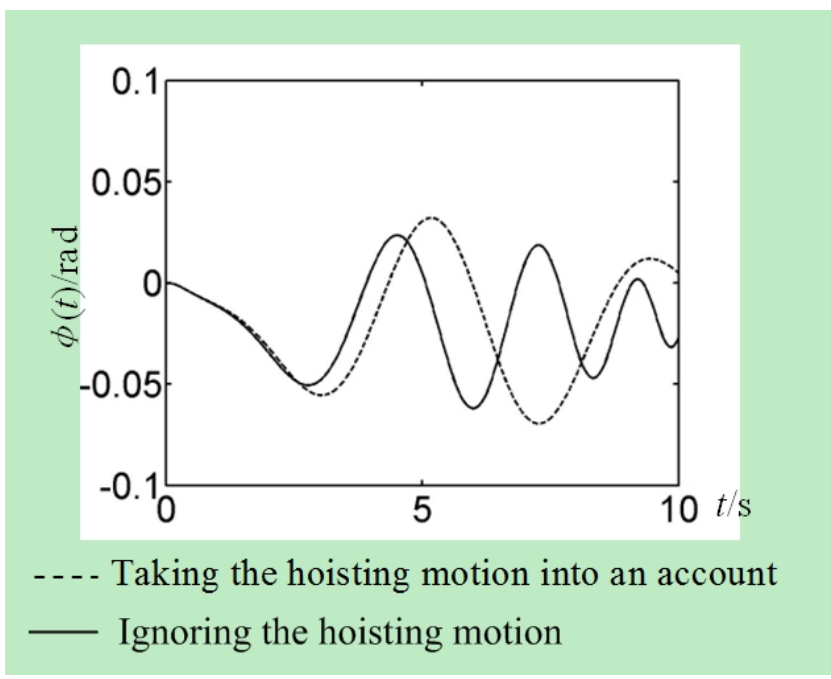

(a) Oscillation angle $\varphi(t)$

Fig. 2. Response curve of the cargo oscillation

From Fig. 2 it can be seen that the swing period is smaller and smaller, the swing amplitude is more and more big with the cargo rising, the center line of the oscillation is tilted. If the hoisting motion is neglected, then the cargo swing period is not changing. During the process of transporting cargo, when hoisting the cargo in the large range, if the hoisting motion is ignored, then there exists the large error of cargo oscillation. So the hoisting motion should be taken into an account. When lowering the cargo, the swing angle response curve of the cargo is shown in Fig. 3. 


\section{Conclusions}

The hoisting motion makes the system become weak damping system. The angle and angular velocity of the cargo swing are the periodic function with variable amplitude and frequency. The amplitude is attenuated or increased in exponential function. Decay or increase of the amplitude in a swing period is $\exp \left(-\pi \xi / \sqrt{\mathrm{g} l-\xi^{2}}\right)$. The frequency is changed with the change of the pendulum length, $\omega_{\mathrm{n}}^{2}=\mathrm{g} / l$. Therefore, the system parameter variation caused by the hoisting and lowering motion should be considered in the design of anti-swing controller.

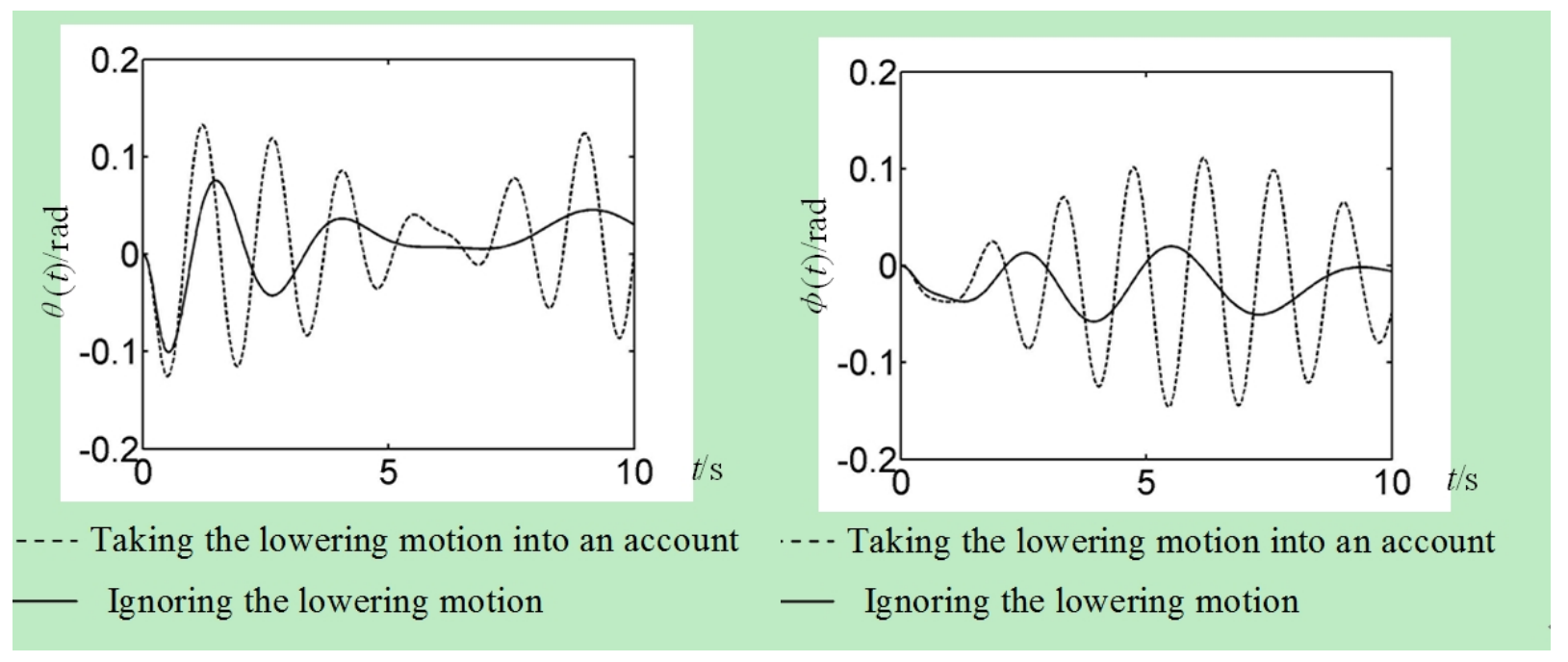

(a) Oscillation angle $\theta(t)$

(b) Oscillation angle $\varphi(t)$

Fig. 3. Response curve of the cargo oscillationc

\section{Acknowledgements}

This work was financially supported by the National Natural Science Foundation of China (Grant No. 51475277).

\section{References}

[1] Bae-Jeong Park,Keum-shik Hong, Chang-Do Huh. Time-efficient input shaping control of container crane systems [A].Proceeding of the 2000 IEEE International Conference on Control Applications[C]. Anchorage, Alaska, USA:2000: 80-85.

[2] James J. Potter, Christopher J. Adams, and William Singhose. A Planar Experimental Remote-Controlled Helicopter with a Suspended Load. IEEE/ASME Transactions on Mechatronic.2014:256-266.

[3] Dongho Kim, Youngjin Park. Tracking control in $x-y$ plane of an offshore container crane. Journal of Vibration and Control. 2015, July 1:1-15.

[4] Daichi Fujioka, Manan Shah, William Singhose. Robustness Analysis of Input-Shaped Model Reference Control on a Double-Pendulum Crane.

[5] Yongchun Fang, Pengcheng Wang, Ning Sun, and Yichun Zhang. Dynamics Analysis and Nonlinear Control of an Offshore Boom Crane. IEEE Transactions on Industrial Electronics, Vol. 61, No. 1, January, 2014:414-427. 
[6] Sami Kiviluoto, Lasse Eriksson, Heikki N. Koivo. Modeling and control of vertical oscillation in overhead cranes.2015 American Control Conference, Palmer House Hilton, Chicago, IL, USA. July 1-3, 2015:1290-1295.

[7] Andreas Rauh, Luise Senkel, Jovanka Gebhardt, Harald Aschemann. Stochastic Methods for the Control of Crane Systems in Marine Applications. 2014 European Control Conference (ECC), June 24-27, 2014, Strasbourg, France,2014: 2998-3003.

[8] Mingxiao Dong. Research on Time-delayed Control Theory and Its Application to Realization of Crane Automation. Xi'an: Xi'an Jiaotong University. 2005, 12, in Chinese.

[9] Mingxiao Dong. Time-delayed filter theory and engineering application. Beijing: Science Press. 2008,3, in Chinese. 